\title{
Maximum Likelihood Estimates for Object Detection Using Multiple Detectors
}

\author{
Magnus Oskarsson and Kalle Åström \\ Centre For Mathematical Sciences, Lund University, Lund, Sweden \\ \{magnuso, kalle\}@maths.lth.se \\ http: //www. maths.1th.se/index.html
}

\begin{abstract}
Object detection in real images has attracted much attention during the last decade. Using machine learning and large databases it is possible to develop detectors for visual categories that have a very high hit-rate, with low false positive rates. In this paper we investigate a general probabilistic framework for context based scene interpretation using multiple detectors. Methods for finding maximum likelihood estimates of scenes given detection results are presented. Although we have investigated how the method works for a specific case, namely for face detection, it is a general method. We show how to combine the results of a number of detectors i.e. face, eye, nose and mouth detectors. The methods have been tested using detectors trained on real images, with promising results.
\end{abstract}

\section{Introduction}

During the last decade much interest in the computer vision community has been put into the areas of object detection, recognition and classification. Using machine learning and large databases it is possible to develop detectors for visual categories such as faces, eyes, cars, bicycles, animals etc.

Many of these detectors work in the following semantic way. A binary classifier is obtained for objects at a predefined scale and position using a large database of positive and negative examples, and machine learning. This classifier is then applied to different parts of the image, i.e. at different positions and different scales. A typical result is shown in Figure 1. Some kind of clustering algorithm is then applied in order to reduce the number of detections that originate from the same object.

In this paper, we discuss (i) how this clustering method can be improved using scene models and (ii) how multiple detectors can be used in conjunction in order to improve the results.

Geometric constraints have been used to improve detection in a number of publications. The constraints can be more or less explicit. In [3] the concept of body plans was used to detect humans and horses. The geometric relationship between parts was explicitly given in the model. In [6] a hierarchical model of components of humans was used in a SVM-framework to detect humans in still images. In [10] probabilities for walking humans were learned with good results. In [5] they use a number of detectors to improve face-detection. Here they optimize over a weighted sum of individual likelihoods from each detector. Context in the form of appearance around object was 
exploited in [4]. In [8] statistics of spatial relationships were learned to improve object detection. For surveys on context and face detection see e.g. [2,13].

In our work we are interested in how one can use scene models and context to, on the one hand, get better detection rates. But another goal is to get higher level information about the detected objects, how they are related to each other geometrically and their properties such as appearance and pose. As will be seen in the experiments on face detection in section 3 one of the biggest gains in combining detectors in a contextual manner is in the precision of detection. We achieve this not by starting with the detections and combine them in some manner, but instead formulate a hypothetical scene and calculate how likely this scene is given the detection result. The problem is then to search the scene space for the most probable scene. By the most probable scene we mean the scene with the highest likelihood given the detections.

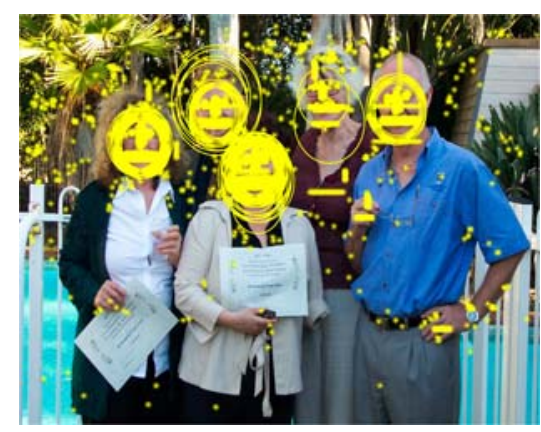

Fig. 1. Typical detection result. Each circle represents a face detected at the center of the circle and the radius corresponds to at which scale the detection was made. At most positions and scales there are no detections.

\section{Scene Modeling and Model Estimation}

\subsection{Scene Modeling}

We assume that the scene contains a number of objects of different types, $\Omega_{i}$, and that for each such object there is a corresponding pose parameter $p$. In the examples below pose includes position in the image and scale. However, depending on the structure of the detector, the pose parameter could include more or fewer parameters. One might, for instance, have a detector that is trained on faces with a given position, distance and orientation relative to the camera. Or the pose parameter could contain shape variational parameters of deforming objects.

For simplicity we assume that the object types form a hierarchy (or a directed acyclic graph). In the simplest case there is only one type of object, e.g. eyes. In the slightly more advanced case there is an hierarchy, e.g. faces are modeled in the scene and then on the next level mouth, nose and eyes pose are conditioned on the pose of the face.

Using images with ground truth position we estimate the probability $P\left(\# \Omega_{i}=n_{i}\right)$, of a scene containing $n_{i}$ objects of type $\Omega_{i}$ at the top level. We then estimate the joint 
probability density function $f\left(p_{i, 1}, \ldots, p_{i, n_{i}}\right)$ of the pose parameters $\left(p_{i, 1}, \ldots, p_{i, n_{i}}\right)$ for these $n_{i}$ objects of type $\Omega_{i}$ at the top level.

We then go on estimating the probability $P\left(\# \Omega_{i j}=n_{i j}\right)$ for object type $\Omega_{i j}$ at the next level of hierarchy and the corresponding pose parameter joint probability density function conditioned on the pose parameter of the ascendent $p_{i}$.

The resulting scene model $x$ is a collection of objects, $\{\Omega\}$, of different types, where each object has a different pose $p$.

Simulation of such models $x$ can be made by sampling the number of objects and their pose parameters at the top level, followed by sampling of objects and poses of objects on lower level in the hierarchy.

Furthermore, for each scene model $x$, the likelihood can be determined as

$$
\begin{gathered}
L(x)=\Pi_{i=1}^{n}\left(P\left(\# \Omega_{i}=n_{i}\right) f\left(p_{i, 1}, \ldots, p_{i, n_{i}}\right) \cdot \ldots\right. \\
\left.\ldots \cdot \Pi_{j=1}^{m_{i}}\left(P\left(\# \Omega_{i j}=n_{i j}\right) f\left(p_{i j, 1}, \ldots, p_{i j, n_{i j}}\right)\right)\right) .
\end{gathered}
$$

\subsection{Detection Modeling}

As was described in the previous section, we have developed a number of detectors for different types of objects. Each such detector is evaluated at a discrete number of positions, $Y=p_{1}, p_{2}, \ldots, p_{N}$, in the corresponding pose space. We assume that the probability of obtaining a detection at pose $p$, given a scene with an object at true pose $\bar{p}$ close to $p$, is only dependent on this true object. This implies that we believe that each object has limited reach, i.e. a detection of an object is plausible only at close distances from the object. At larger distances there is still a possibility of 'false' detections, which we assume to be constant in the pose space.

In the experiments we have furthermore assumed a kind of stationarity in this respect. We have used pose spaces such as (position+scale) and have assumed that the probability depends on relative position and relative scale between the detected pose $p$ and the actual pose $\bar{p}$. This probability

\section{$P_{\text {detect }}($ detection at pose $p \mid$ closest pose is $\bar{p})$}

is estimated from databases with objects of known pose.

Each studied image is analyzed by running all of the detectors of type $\Omega_{i j}$ at all sampled positions $Y_{i j}$. The result $y$ is typically a small number of positive detections of different types and a large number of non-detections of different types. Such a result $y$ is shown in Figure 1, where each circle represents a face detected at the center of the circle and the radius corresponds to at which scale the detection was made. One may view $y$ as a large boolean vector of length $N=\sum_{i j} N_{i j}$.

For each such detection result it is possible to determine the likelihood of the detection given a scene model $x$,

$$
P(y \mid x)=\Pi_{k}^{N} P_{\text {detect }}\left(y_{i} \mid x\right) .
$$




\subsection{Scene Model Estimation from Ensemble Detections}

Assume that we have an image, on which we have run multiple detectors and have obtained a detection result $y$. The scene model estimation is then given by maximum aposteriori estimation

$$
\tilde{x}=\arg \max _{x} P(y \mid x) f(x) .
$$

One problem with this approach is that the prior $f(x)$ for different scene models vary in magnitude with different number of degrees of freedom for the scene model observation space, i.e. scene models containing many objects have probability density function values several magnitudes lower than those of few objects.

For an example of scene modeling see section 3.2.

Finding the optimum over all possible scene models $x$ is a large optimization problem. The idea here is not that the scene model estimation should be performed by exhaustive search of this space. The main point is that the likelihood serves as a basis for comparing and choosing between different results obtained by heuristics, e.g. clustering of detection results. Using this model we hope to obtain better results than ad hoc methods which are used today, e.g. [3,5].

\section{Experiments on Face Detection}

\subsection{Detection Probabilities}

In these experiments we have used four detectors for faces, eyes, noses and mouths. Each detector has been trained on different positions and scales, i.e. the pose space is three dimensional $(x, y, s)$. The detectors are based on boosting as described in [12] with cascades.

Typically the detection pose set, as illustrated in Figure 2, has coarser sampling in space $(x, y)$ for coarser (larger) scales.

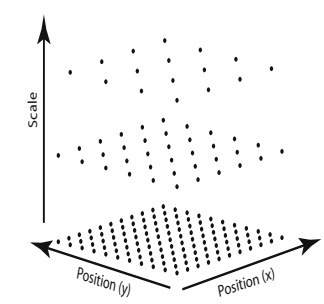

Fig. 2. Object detection is performed at a discrete set of pose points as illustrated in the figure

A typical detection (before clustering) is shown in Figure 1.

As can be expected when detecting an object at pose $p_{m}$, the detector will return true for many tested poses close to that position. By studying 697 images containing 
995 faces with known poses, we estimate the probability of detecting an object at pose $p_{d}$ when the true pose is $p_{m}$. Here only the relative pose is used

$$
p_{\text {rel }}=\left(\begin{array}{c}
\left(p_{d, x}-p_{m, x}\right) / p_{m, s} \\
\left(p_{d, y}-p_{m, y}\right) / p_{m, s} \\
\log \left(p_{d, s} / p_{m, s}\right)
\end{array}\right)
$$

Notice that the relative pose $p_{\text {rel }}$ is invariant under a common translation and scale. Notice also that $p_{d}=p_{m}$ implies that $p_{r e l}=\mathbf{0}$. For each type of object, (face, eyes, nose and mouth), the probability of detection

$$
P_{\text {det }}\left(p_{\text {rel }}\right)=P\left(\text { detect at relative pose } p_{\text {rel }}\right)
$$

is estimated using a three-dimensional histogram. The result is shown in Figure 3.

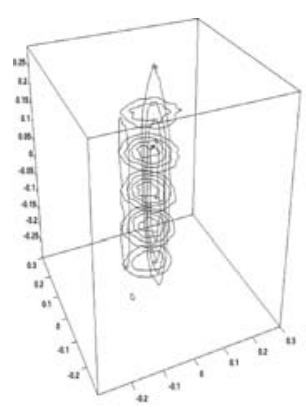

(a)

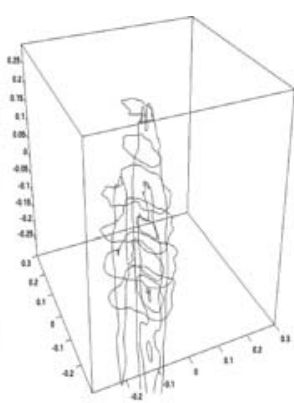

(b)

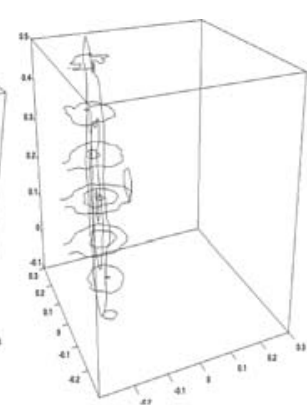

(c)

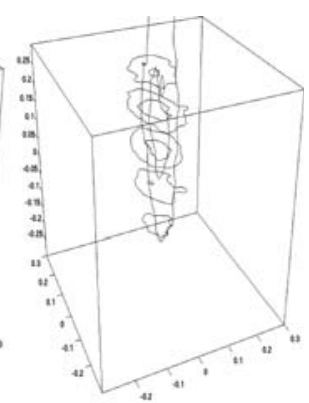

(d)

Fig. 3. Estimated detection probability densities as a function of relative pose for (a) faces, (b) eyes, (c) noses and (d) mouths. Here the position are on the $\mathrm{x}$ - and $\mathrm{y}$-axes and the scale is on the $\mathrm{z}$-axis. Notice the bias in nose position. This is due to a difference in nose center definition between the training of the detector and the ground truth modeling made here.

We approximate these detection probabilities as scaled Gaussian functions, i.e.

$$
P_{\text {det }}=a \frac{1}{(2 \pi)^{3 / 2} \sqrt{|\Sigma|}} e^{\left(p_{r e l}-m\right)^{T} \Sigma^{-1}\left(p_{\text {rel }}-m\right) / 2},
$$

with different parameters $(a, m, \Sigma)$ for each detector. Notice that there might be a tradeoff here between having a very specific detector with high detection probability only in a small region, or a very unspecific detector with a broad detector response and perhaps not so high detection probability. As can be seen in the examples the detectors are quite specific in position, but not so specific in terms of relative scale.

\subsection{Scene Modeling}

In this experiments we modeled the scene as having a number of faces, each of which contained two eyes, one nose and one mouth. We estimated model probabilities from 


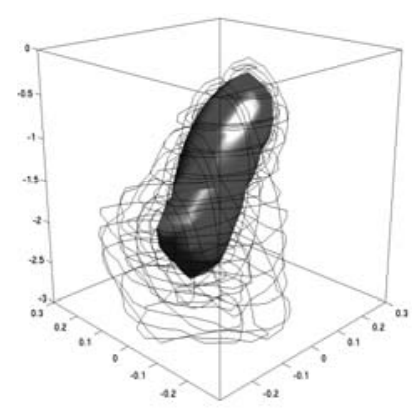

(a)

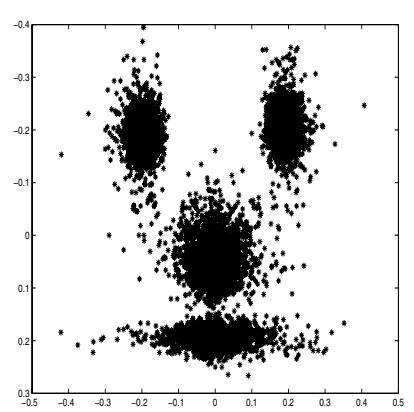

(b)

Fig. 4. (a) Estimated probability density function for faces in pose space. Notice that the ycoordinate is typically higher for smaller faces (lower scales) and that there is larger variations in position (both $\mathrm{x}$ and $\mathrm{y}$ ) for smaller faces. Larger faces tend to be more centrally positioned and with smaller variance in position. (b) The distribution of eyes, noses and mouths relative to the face poses in the database.

2268 images. These contained different number of faces. In total there were 2551 faces in these images. Images come in different sizes and shapes. We adopted a scene coordinate model with origin in the middle of the image and base scale equal to the square root of the image area in pixels. We represented image pose in this space with scale coordinate equal to the logarithm of the face width divided by the base scale. Figure 4 illustrates the estimated probability density function of a random face in this pose space.

In the experiment the following approximations were made. The positions of faces in a random image were assumed to be independently distributed according to Figure 4a with the addition that no two faces were allowed to spatially overlap more than 30 percent. This is not entirely realistic, since an image with many faces will probably be biased towards smaller scales.

Furthermore we assume that the relative pose of the two eyes, the nose and the mouth can be approximated by Gaussian distributions. Figure $4 \mathrm{~b}$ shows the distribution of such facial positions relative to the face in the 2551 faces in the database.

\subsection{Optimizing Likelihood}

A two step maximization of the Likelihood was made:

$$
\max _{\text {scene }} \log P(\text { detection } \mid \text { scene }) \text {. }
$$

Initial guesses for scenes were found from detected faces, noses, and mouths. From each detected feature a face with two eyes, a nose and a mouth was estimated using estimated relative mean positions. The procedure works in the following way,

1. Randomly select a feature from all detected features.

2. From this feature estimate a whole face. Add this to the current scene.

3. Calculate the likelihood for the new test-scene, and if this is greater than the current maximum, this is the new optimum. 
The procedure starts with an empty scene and is repeated for a number of iterations. The best scene is then kept and the whole procedure is done all over again but starting with the current best scene instead of an empty scene. This procedure is repeated for a number of iterations, i.e. as many times as the maximum number of faces to be expected. This means that the number of faces is chosen automatically, the only restriction being that the maximum number of faces that can be found is limited by the number of iterations.

Given an initial scene we can then optimize the scene by moving each individual feature in the scene, i.e. the faces, eyes, noses and mouths, in position and scale. This is done by a simple neighborhood search.

\subsection{Results}

Using the estimated image and detector probabilities, we have tested the detection system on a number of images. For these images we obtained ground truth by manually marking positions in the images. A typical detection result can be seen in Figure 5. The database used for testing consisted of color images of groups of people collected from the Internet, with one to fifteen fronto-parallel faces. In total we used 300 images with a total of 1437 faces. This database is completely different from the database used for estimating all the detection and model probabilities, and can be acquired from the authors upon request. The results from the four detectors were collected and the maximum likelihood estimate of the scene was calculated according to section 3.3. The recall and precision were calculated for the face, nose and mouth detectors as well as for the combined result. The recall or hit-rate is defined as the total number of correct detections divided by the total number of faces in the images. The precision is defined as the number of correct detections divided by the total number of detections. The result can be seen in Table 1 . Notice that only a single precision-recall (P/R) value is given. As our method is based on finding a maximum likelihood estimate, the result is based on the detection results from the different detectors, and the estimated detector statistics. This means that it is not straight-forward to to get a P/R curve by tuning a detection rate. By tuning the different detectors in different ways one would probably get different $P / R$ curves depending on which detector one changed. One way to get a P/R curve might be to change the detector statistics, but as there is no detection threshold there are many parameters which one can vary. Also since these parameters are estimated and hopefully close to the true parameters, changing them would change the model, and the final detection would then not be the maximum likelihood estimate of the scene. As can be seen, the precision of the mouth and nose detectors aren't very high. The eye detector is not included in the table, but its precision is even lower. The precision of the face detector is quite high, but the recall is not very high. The missed faces are in most cases

Table 1. Recall and precision for the different detectors as well as for the maximum likelihood estimate

\begin{tabular}{c|c|c|c|c} 
& Nose & Mouth & Face & Combined \\
\hline Precision & 0.45 & 0.50 & 0.97 & 1.00 \\
\hline Recall & 0.81 & 0.79 & 0.75 & 0.84
\end{tabular}



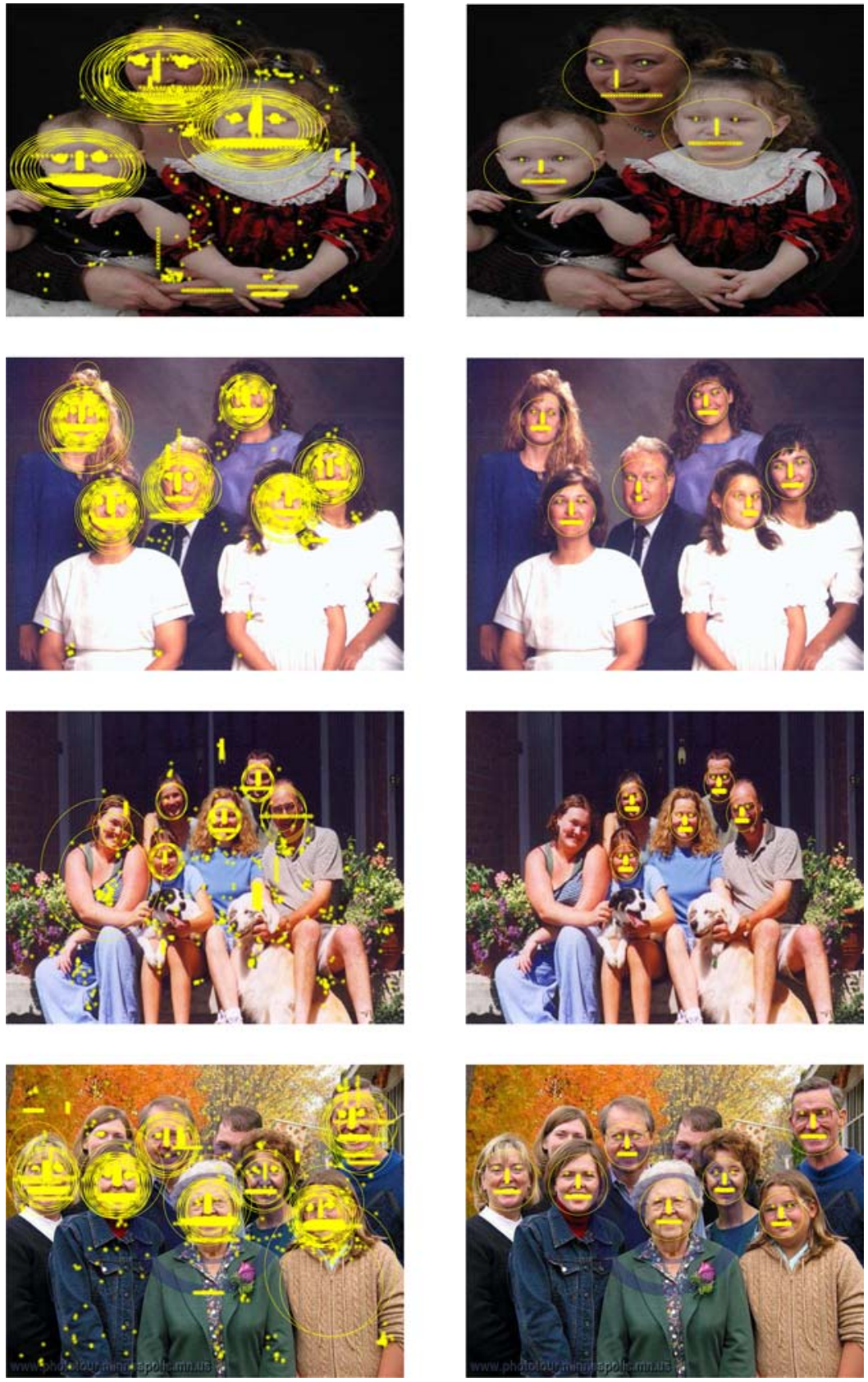

Fig. 5. On the left hand side images the initial unclustered detection results on a number of images is shown. The face detections are indicated by blue circles, the eyes by magenta stars, the mouths by red horizontal lines and the noses by yellow vertical lines. The sizes indicate the scale of the detections. On the right hand side the maximum likelihood estimates of the scenes are shown. 
children or older people which were scarce in the training database for the detectors. One can see that the precision of the combined detector is very high; there were only three false positive faces in all the tested images.

\section{Conclusions}

In this paper we have investigated a probabilistic framework for context based scene interpretation using multiple detectors. Methods for finding maximum likelihood estimates of scenes given detection results were presented. The benefits of optimizing a scene model given a detection result is manifold. Firstly we get a clustering method that gives valid results in the sense that they adhere to a given real world model. Secondly we get higher recall rates than just using the individual detectors. But the most important gain is that we get a better understanding of the detected scene and the objects in it. This leads to both higher precision detections and the possibility to infer properties of the imaged object such as e.g. pose. One draw-back is of course that we need to use more computational effort compared to just using one specific mid-level detector.

\section{References}

1. D. Crandall, P. Felzenszwalb, and D. Huttenlocher. Spatial priors for part-based recognition using statistical models. In Proc. Conf. Computer Vision and Pattern Recognition, San Diego, USA, pages I: 10-17, 2005.

2. I. L. Dryden, K. V. Mardia, and A. N. Walder. Review of the use of context in statistical image analysis. J. of Applied Statistics, 24(5):513-538, 1997.

3. D. Forsyth and M. Fleck. Body plans. In CVPR, Puerto Rico, USA, pages 678-683, 1997.

4. H. Kruppa and B. Schiele. Using local context to improve face detection. In BMVC, Norwich, England, pages 3-12, 2003.

5. C. Mikolajczyk, K.and Schmid and A. Zisserman. Human detection based on a probabilistic assembly of robust part detectors. In ECCV, Prague, Czech Republic, 2004.

6. A. Mohan, C. Papageorgiou, and T. Poggio. Example-based object detection in images by components. IEEE Trans. Pattern Analysis and Machine Intelligence, 23(4):349-361, 2001.

7. S.J.D. Prince, J.H. Elder, Y. Hou, M. Sizintsev, and Y. Olevskiy. Statistical cue integration for foveated wide-field surveillance. In Proc. Conf. Computer Vision and Pattern Recognition, San Diego, USA, pages II: 603-610, 2005.

8. H. Schneiderman. Learning statistical structure for object detection. In CAIP, Groningen, Netherlands, pages 434-441, 2003.

9. H. Schneiderman and T. Kanade. Object detection using the statistics of parts. Int. Journal of Computer Vision, 56(3):151-177, February 2004.

10. H. Sidenbladh and M. Black. Learning image statistics for bayesian tracking. In ICCV, vancouver, canada, pages 709-716, 2001.

11. A. Torralba. Contextual priming for object detection. Int. Journal of Computer Vision, 53(2):169-191, July 2003.

12. P. Viola and M. Jones. Rapid object detection using a boosted cascade of simple features. In Proc. Conf. Computer Vision and Pattern Recognition. IEEE Computer Society Press, 2001.

13. M.H. Yang, D.J. Kriegman, and N. Ahuja. Detecting faces in images: A survey. IEEE Trans. Pattern Analysis and Machine Intelligence, 24(1):34-58, January 2002. 